\title{
Phenotypic spectrum of TGFB3 disease-causing variants in a Dutch-French cohort and first report of a homozygous patient
}

\section{Running title: Phenotypic spectrum of TGFB3 variants}

Luisa Marsili ${ }^{1,2 \#}$, Eline Overwater ${ }^{2,3}$, Nadine Hanna ${ }^{4,5}$, Geneviève Baujat ${ }^{6}$, Marieke J.H. Baars ${ }^{3}$, Catherine Boileau ${ }^{4,5,7}$, Dominique Bonneau ${ }^{8}$, Anne Claire Brehin ${ }^{9}$, Yline Capri ${ }^{10}$, Ho Y. Cheung ${ }^{3}$, Eelco Dulfer ${ }^{11}$, Marion Gerard ${ }^{12}$, Laurent Gouya ${ }^{7}$, Yvonne Hilhorst-Hofstee ${ }^{13}$, Arjan C. Houweling ${ }^{2}$, Bertrand Isidor ${ }^{14}$, Lauriane Le Gloan ${ }^{15}$, Leonie A. Menke ${ }^{3}$, Sylvie Odent ${ }^{16}$, Fanny Morice-Picard ${ }^{17}$, Clemence Vanlerberghe ${ }^{1}$, Els Voorhoeve ${ }^{2}$, J. Peter van Tintelen ${ }^{3,18}$, Alessandra Maugeri²* and Pauline Arnaud ${ }^{4,5 *}$

${ }^{1}$ CHU Lille, Clinique de Génétique, 59000, Lille, France.

${ }^{2}$ Department of Clinical Genetics, Amsterdam UMC, Vrije Universiteit Amsterdam, Amsterdam, The Netherlands. ${ }^{3}$ Department of Clinical Genetics, Amsterdam UMC, University of Amsterdam, Amsterdam, The Netherlands. ${ }^{4}$ Département de Génétique, Assistance Publique-Hôpitaux de Paris, Hôpital Bichat, Paris, France.

${ }^{5}$ Laboratory for Vascular Translational Science, INSERM U1148, Université Paris Diderot, Hôpital Bichat, Paris, France.

${ }^{6}$ Service de Génétique, INSERM U781, Hôpital Necker-Enfants Malades, Institut Imagine, University SorbonneParis-Cité, Paris, France.

${ }^{7}$ Centre de référence pour le syndrome de Marfan et apparentés, Assistance Publique-Hôpitaux de Paris, Hôpital Bichat, Faculté Paris Diderot, LVTS INSERM U 1148, Paris, France.

${ }^{8}$ Service de génétique, CHU d'Anger, Angers, France.

${ }^{9}$ Department of Genetics, Normandy Centre for Genomic and Personalized Medicine, Normandie University, UNIROUEN, Inserm U1245 and Rouen University Hospital, F 76000, Rouen, France.

${ }^{10}$ Department of Genetics, APHP-Robert DEBRE University Hospital, Denis Diderot School of Medicine, Paris University, France.

${ }^{11}$ Department of Medical Genetics, University Medical Center Groningen, 9713 GZ Groningen, the Netherlands.

${ }^{12}$ Service de Génétique, CHU Caen, Caen, France.

${ }^{13}$ Department of Clinical Genetics, Leiden University Medical Center, Leiden, the Netherlands. 
${ }^{14}$ CHU Nantes, Service de Génétique Médicale, Nantes, France.

${ }^{15} \mathrm{CHU}$ Nantes, Clinique cardiologique et des maladies vasculaires, Nantes, France.

${ }^{16} \mathrm{CHU}$ de Rennes, service de Génétique clinique, Université Rennes, CNRS UMR6290 IGDR (Institut de Génétique et Développement de Rennes), Rennes, France.

${ }^{17}$ Service de dermatologie, Unité de dermato-pédiatrie du CHU de Bordeaux, Bordeaux, France.

${ }^{18}$ Utrecht University Medical Center, Utrecht University, department of Genetics, Utrecht, the Netherlands.

*Contributed equally

\#Corresponding author:

L. Marsili, CHU Lille, Clinique de Génétique, Hôpital Jeanne de Flandre, Avenue Eugène Avinée 59037, Lille, France. Tel: +33(0)320444911, Fax: +33(0)320444901. Email address: luisa.marsili@chru-lille.fr

\section{CONFLICT OF INTEREST}

None.

\section{DATA AVAILABILITY STATEMENT}

Data supporting the findings of this study are available from the corresponding author. 


\section{ABSTRACT}

Disease-causing variants in TGFB3 cause an autosomal dominant connective tissue disorder which is hard to phenotypically delineate because of the small number of identified cases. The purpose of this retrospective cross-sectional multicentre study is to elucidate the genotype and phenotype in an international cohort of TGFB3 patients.

Eleven (8 novel) TGFB3 disease-causing variants were identified in 32 patients (17 families). Aortic root dilatation and mitral valve disease represented the most common cardiovascular findings, reported in $29 \%$ and $32 \%$ of patients, respectively. Dissection involving distal aortic segments occurred in two patients at age 50 and 52 years. A high frequency of systemic features (65\% high-arched palate, 63\% arachnodactyly, 57\% pectus deformity, 52\% joint hypermobility) was observed. In familial cases, incomplete penetrance and variable clinical expressivity were noted. Our cohort included the first described homozygous patient, who presented with a more severe phenotype compared to her heterozygous relatives.

In conclusion, TGFB3 variants were associated with a high percentage of systemic features and aortic disease (dilatation/dissection) in 35\% of patients. No deaths occurred from cardiovascular events or pregnancy-related complications. Nevertheless, homozygosity may be driving a more severe phenotype.

\section{KEY WORDS}

TGFB3, transforming growth factor beta 3, connective tissue disorder, aortic dilatation, aortic dissection, Loeys-Dietz syndrome 


\section{INTRODUCTION}

A heritable connective tissue disorder due to TGFB3 variants was first described in 2013 and listed in OMIM as Loeys-Dietz syndrome-5 (\#615582), although controversy exists regarding this nomenclature. ${ }^{1,2}$ Its clinical manifestations mainly involve the skeletal, ocular and cardiovascular systems.

Given the rarity of the disorder, with no more than 50 cases described so far, a precise delineation of its phenotype is yet to be determined. ${ }^{3-10}$

Here, we report the clinical and genetic findings of 32 patients from 17 families, and we give an overview of all reported TGFB3 disease-causing variants. Our aim is to achieve a better understanding of the phenotype related to TGFB3 disease-causing variants.

\section{METHODS}

Patients were identified through the diagnostic laboratories at the Department of Clinical Genetics, Amsterdam UMC, Vrije Universiteit Amsterdam, the Netherlands, and at the Département de Génétique, Hôpital Bichat, Paris, France (Supporting Information). Referring physicians were invited to participate in this study by a written invitation and were mailed questionnaires in order to retrospectively collect clinical data. The data collection period was from April 2018 to May 2019. All genetic and clinical data were acquired during standard patient care. Informed consent for publication was obtained from all patients, or their legal representatives, in accordance with the Declaration of Helsinki and national legal regulations. 
In order to obtain an overview of all known disease-causing TGFB3 variants, databases and previously published articles were consulted (Supporting Information).

\section{RESULTS}

\section{TGFB3 variants}

Eleven (8 novel) variants were detected in our cohort: 7 missense, one nonsense, and three splice site variants (Table 1 and Table 2). Four variants (36\%) were located in the RKKR motif. According to the AMCG criteria, ${ }^{10}$ all variants are classified as pathogenic or likely pathogenic except for p.(Asp303Ala) and p.(Ser359Arg) that are variants of unknown significance (Supporting Information). Twenty-six other variants were extrapolated from databases and literature (Table 2).

\section{Clinical data}

Our cohort consisted of 32 TGFB3 patients (56\% males) from 17 families. Mean age at last evaluation was 32 years (range 4-60 years).

Aortic dilatation or dissection was observed in 11/31 (35\%) of patients and exhibited age-related penetrance (Figure 1). Aortic root dilatation occurred in 9/31 (29\%) patients, and was associated with dilatation of distal aorta in three patients. Two patients presented with distal aortic dilatation in the absence of aortic root dilatation. Dissection of the aorta occurred in two patients at age 50 and 52. Two patients underwent preventive aortic surgery, both after age 40. Two patients displayed arterial tortuosity. Mitral valve disease (prolapse and/or insufficiency) and aortic valve insufficiency were observed in 9/28 (32\%) and 2/28 (7\%) of 
patients, respectively. Neither atrial/ventricular septal defects nor atrioventricular block were noted. Varices of lower limbs were seen in 5/24 (21\%) of patients.

Average height was $+1.3 \mathrm{SD}$ (range $-1 \mathrm{SD}$ to $+4 \mathrm{SD}$ ). The most common systemic signs were high-arched palate (65\%), arachnodactyly (63\%), pes planus (63\%), pectus deformity (57\%), and joint hypermobility (52\%), long face (42\%), downslanting palpebral fissures (39\%), bifid uvula (38\%), increased arm span (36\%), easy bruising (34\%), thin translucent skin (32\%), myopia (33\%), hypertelorism (32\%), and scoliosis (30\%). Some of the features previously associated with variants in TGFB3 were not observed (brachycephaly, wide face, ectopia lentis, scapulae alata, cervical spine instability, contractures of fingers, palmar flexion, transient postnatal pes adductus, low birth weight), or were observed in $10 \%$ of patients or less (metopic ridge, midface hypoplasia, smooth philtrum, exotropia, ptosis, cerebral hemorrhage, osteoporosis, camptodactyly of toes, hiatal hernia, osteo-arthritis, spondylolisthesis, bilateral coxa valga, congenital hypotonia, delayed motor development, autoimmune disease).

No major vascular or visceral complication occurred in 18 pregnancies (six women).

\section{Homozygous patient}

Patient \#28 was the first child of consanguineous parents of Moroccan origin. She was born at 38/2 weeks of gestation. Birth weight was 2930 grams. Cleft palate, umbilical hernia, and joint contractures (involving elbows, knees and feet, which improved significantly over time) were diagnosed shortly after birth. Arachnodactyly and dysmorphic craniofacial features, including micrognathia and low-set ears, were also observed in the neonatal period. 
She was later diagnosed with a left-sided bochdalek hernia and developed pectus excavatum, L5-S1 spondylolisthesis, scoliosis, severe myopia, and dilatation of the aortic root (Z-score 2.14 at age 17 years). She received medroxyprogesterone and ethinylestradiol for anti-growth purposes, and she was treated by long-term nocturnal gastrostomy feedings to improve her nutritional status. Both motor and cognitive developments were normal. Genetic testing revealed a homozygous c.1081-2A>T TGFB3 variant (Supporting Information). Both parents (patients \#31 and \#32), a brother (patient \#29), and a sister (patient \#30) were heterozygous and were less severely affected. Another sibling didn’t carry the variant and displayed no sign of the disease.

\section{DISCUSSION}

Our findings are largely in line with the most comprehensive report on TGFB3 variants published before. ${ }^{8}$ Aortic disease (dilatation or dissection) affected $35 \%$ of patients showing age-related penetrance (Figure 1). No aortic dilatation occurred before the age of 14 years. Arterial dissections occurred at dilated aortic segments after the age of 50 years in two patients who displayed marked systemic features. No deaths related to cardiovascular or pregnancy complications were observed. Men were more likely to be diagnosed with aortic disease, but difference between men and women was not significant. Arterial tortuosity was observed in two patients. Two patients were diagnosed with dural ectasia, a clinical feature which was not previously described in association with TGFB3 variants. As TGFB3 patients are not systematically screened for dural ectasia, whether this is a coincidental finding or an underestimated clinical feature remains unclear. 
Compared to Schepers et al, ${ }^{8}$ we identified a higher rate of downslanting palpebral fissures (39\% vs. 15\%), and a lower rate of joint hypermobility (52\% vs. 80\%), scoliosis (30\% vs. 69\%), and osteoarthritis (8\% vs. 46\%). Clubfoot was reported by Schepers and colleagues in $25 \%$ (3/12) of patients. ${ }^{8}$ Two (7\%) related patients in our cohort presented with clubfoot. However, clubfoot in this family may not be related to the TGFB3 variant since a family member, who did not have the TFGB3 variant, exhibited clubfoot in the absence of any other systemic feature (cardiac investigation not performed). Differences in results between previous studies and ours may reflect differences in clinical assessment and/or in reason of referral for genetic testing.

Consistently with data from literature and databases, in our cohort we observed missense, splice site, and truncating variants spanning the entire gene, with a mutation hotspot in the RKKR motif.

Finally, we described the first homozygous TGFB3 patient, who presented with aortic dilatation at a younger age and more marked systemic features, compared to her heterozygous relatives.

\section{Limitations of the study}

This study is limited by small patient numbers, relatively young age of patients, possible ascertainment bias due to recruitment, and retrospective nature of the data.

\section{CONCLUSION}


No more than 50 patients carrying disease-causing TGFB3 variants have been reported so far. ${ }^{1,3-9}$ Here, we described genetic and clinical data from 32 TGFB3 patients from 17 families including the first homozygous individual.

In our cohort, variants in TGFB3 were associated with a high frequency of systemic features. Aortic disease (dilatation or dissection) was revealed in 35\% of patients, but no increased overall mortality nor pregnancy complications were observed. Nevertheless, homozygosity might be associated with a more severe phenotype.

Finally, we suggest that regular surveillance of distal aorta is appropriate in TGFB3 patients. 


\section{Reference list}

1. Rienhoff HY Jr, Yeo CY, Morissette R, Khrebtukova I, Melnick J, Luo S, Leng N, Kim YJ, Schroth G, Westwick J, Vogel H, McDonnell N, Hall JG, Whitman M. A mutation in TGFB3 associated with a syndrome of low muscle mass, growth retardation, distal arthrogryposis and clinical features overlapping with Marfan and Loeys-Dietz syndrome. Am J Med Genet A. 2013 Aug;161A(8):2040-6. doi: 10.1002/ajmg.a.36056.

2. Pyeritz R, Jondeau G, Moran R, De Backer J, Arbustini E, De Paepe A, Milewicz D. LoeysDietz syndrome is a specific phenotype and not a concomitant of any mutation in a gene involved in TGF- $\beta$ signaling. Genet Med. 2014 Aug;16(8):641-2. doi: 10.1038/gim.2014.63.

3. Bertoli-Avella AM, Gillis E, Morisaki H, Verhagen JMA, de Graaf BM, van de Beek G, Gallo E, Kruithof BPT, Venselaar H, Myers LA, Laga S, Doyle AJ, Oswald G, van Cappellen GWA, Yamanaka I, van der Helm RM, Beverloo B, de Klein A, Pardo L, Lammens M, Evers C, Devriendt K, Dumoulein M, Timmermans J, Bruggenwirth HT, Verheijen F, Rodrigus I, Baynam G, Kempers M, Saenen J, Van Craenenbroeck EM, Minatoya K, Matsukawa R, Tsukube T, Kubo N, Hofstra R, Goumans MJ, Bekkers JA, Roos-Hesselink JW, van de Laar IMBH, Dietz HC, Van Laer L, Morisaki T, Wessels MW, Loeys BL. Mutations in a TGF- $\beta$ ligand, TGFB3, cause syndromic aortic aneurysms and dissections. J Am Coll Cardiol. 2015 Apr 7;65(13):1324-1336. doi: 10.1016/j.jacc.2015.01.040.

4. Kim YM, Lee YJ, Park JH, Lee HD, Cheon CK, Kim SY, Hwang JY, Jang JH, Yoo HW. High diagnostic yield of clinically unidentifiable syndromic growth disorders by targeted exome sequencing. Clin Genet. 2017 Dec;92(6):594-605. doi: 10.1111/cge.13038.

5. Kuechler A, Altmüller J, Nürnberg P, Kotthoff S, Kubisch C, Borck G. Exome sequencing identifies a novel heterozygous TGFB3 mutation in a disorder overlapping with Marfan and Loeys-Dietz syndrome. Mol Cell Probes. 2015 Oct;29(5):330-4. doi: 10.1016/j.mcp.2015.07.003.

6. Matyas G, Naef P, Tollens M, Oexle K. De novo mutation of the latency-associated peptide domain of TGFB3 in a patient with overgrowth and Loeys-Dietz syndrome features. Am J Med Genet A. 2014 Aug;164A(8):2141-3. doi: 10.1002/ajmg.a.36593.

7. Overwater E, Marsili L, Baars MJH, Baas AF, van de Beek I, Dulfer E, van Hagen JM, HilhorstHofstee Y, Kempers M, Krapels IP, Menke LA, Verhagen JMA, Yeung KK, Zwijnenburg PJG, Groenink M, van Rijn P, Weiss MM, Voorhoeve E, van Tintelen JP, Houweling AC, Maugeri A. Results of next-generation sequencing gene panel diagnostics including copy-number variation analysis in 810 patients suspected of heritable thoracic aortic disorders. Hum Mutat. 2018 Sep;39(9):1173-1192. doi: 10.1002/humu.23565.

8. Schepers D, Tortora G, Morisaki H, MacCarrick G, Lindsay M, Liang D, Mehta SG, Hague J, Verhagen J, van de Laar I, Wessels M, Detisch Y, van Haelst M, Baas A, Lichtenbelt K, Braun K, van der Linde D, Roos-Hesselink J, McGillivray G, Meester J, Maystadt I, Coucke P, ElKhoury E, Parkash S, Diness B, Risom L, Scurr I, Hilhorst-Hofstee Y, Morisaki T, Richer J, Désir J, Kempers M, Rideout AL, Horne G, Bennett C, Rahikkala E, Vandeweyer G, Alaerts M, Verstraeten A, Dietz H, Van Laer L, Loeys B. A mutation update on the LDS-associated 
genes TGFB2/3 and SMAD2/3. Hum Mutat. 2018 May;39(5):621-634. doi: 10.1002/humu.23407.

9. Ziganshin BA, Bailey AE, Coons C, Dykas D, Charilaou P, Tanriverdi LH, Liu L, Tranquilli M, Bale AE, Elefteriades JA. Routine Genetic Testing for Thoracic Aortic Aneurysm and Dissection in a Clinical Setting. Ann Thorac Surg. 2015 Nov;100(5):1604-11. doi: 10.1016/j.athoracsur.2015.04.106.

10. Renard M, Francis C, Ghosh R, Scott AF, Witmer PD, Adès LC, Andelfinger GU, Arnaud P, Boileau C, Callewaert BL, Guo D, Hanna N, Lindsay ME, Morisaki H, Morisaki T, Pachter N, Robert L, Van Laer L, Dietz HC, Loeys BL, Milewicz DM, De Backer J. Clinical Validity of Genes for Heritable Thoracic Aortic Aneurysm and Dissection. J Am Coll Cardiol. 2018 Aug 7;72(6):605-615. doi: 10.1016/j.jacc.2018.04.089.

11. Richards S, Aziz N, Bale S, Bick D, Das S, Gastier-Foster J, Grody WW, Hegde M, Lyon E, Spector E, Voelkerding K, Rehm HL; ACMG Laboratory Quality Assurance Committee. Standards and guidelines for the interpretation of sequence variants: a joint consensus recommendation of the American College of Medical Genetics and Genomics and the Association for Molecular Pathology. Genet Med. 2015 May;17(5):405-24. doi: 10.1038/gim.2015.30. 


\section{Figure Legend}

Figure 1

Inverted Kaplan-Meier curve indicating the age-related penetrance of aortic disease, with age at first aortic event (dilatation/dissection/surgery) as the survival variable. 
Table 1. Summary of the relevant clinical and genetic features of patients in our cohort

\begin{tabular}{|c|c|c|c|c|c|c|c|}
\hline ID & Rel & $\begin{array}{l}\text { Sex, } \\
\text { age }^{\dagger}\end{array}$ & Cardiovascular feature(s) & Systemic feature(s) & $\mathrm{FH}$ & Variant & Effect ${ }^{\ddagger}$ \\
\hline 1 & IC & q, 52 & $\begin{array}{l}\text { Dissection (AA, 50y, aortic diameter at dissection } \\
\text { appr. } 63 \mathrm{~mm} \text { ), dilatation (iliac artery, } 51 \mathrm{y} \text { ), } \\
\text { tortuosity (internal carotid and vertebral arteries), } \\
\text { varices }\end{array}$ & $\begin{array}{l}\text { Height }+0.5 \mathrm{SD} \text {, retrognathia, myopia (-7 dioptres), DPF, crowded teeth, thin upper lip, pointed } \\
\text { nose, kyphosis, color blindness, symptomatic hypokalemia }(52 \mathrm{y})\end{array}$ & + & $\begin{array}{l}\text { c.170dup } \\
\text { p.(Glu58*) }\end{array}$ & $\mathrm{P}(\mathrm{Ic})$ \\
\hline 2 & $\mathrm{Si}$ & +50 & Tortuosity (internal carotid and vertebral arteries) & Hypertelorism, PE, JH, Ara, PP, EB & + & $\begin{array}{l}\text { c.170dup } \\
\text { p.(Glu58*) }\end{array}$ & $\mathrm{P}(\mathrm{Ic})$ \\
\hline 3 & IC & q, 51 & Dilatation (thoracic aorta, $48 \mathrm{y}$ ), varices (17y) & $\begin{array}{l}\text { Height + } 4 \text { SD, retrognathia, long face, smooth philtrum, BU, HAP, asymmetric pectus, bilateral } \\
\text { coxa valga, Ara, PP, EB }\end{array}$ & + & $\begin{array}{l}\text { c. } 463 C>T \\
\text { p. }(\operatorname{Arg} 155 \operatorname{Trp})\end{array}$ & LP (II) \\
\hline 4 & IC & +, 16 & None & Height +1 SD, HAP, PE, breast asymmetry, scoliosis, Ara & $?$ & $\begin{array}{l}\text { c. } 463 C>T \\
\text { p. }(\operatorname{Arg} 155 \operatorname{Trp})\end{array}$ & LP (II) \\
\hline 5 & IC & $0^{\lambda}, 21$ & Dilatation (AoR, 16y) & $\begin{array}{l}\text { Height +3.5 DS, dolichocephaly, retrognathia, myopia, DPF, HAP, cleft palate, PC, } \\
\text { kyphoscoliosis, JH, Ara, PP, sternoclavicular subluxation, SS, autoimmune thrombocytopenia, } \\
\text { chronic diarrhea }\end{array}$ & ? & $\begin{array}{l}\text { p. } 516+1 \mathrm{G}>\mathrm{A} \\
\text { p. ? }\end{array}$ & LP (I) \\
\hline 6 & IC & 0,50 & $\begin{array}{l}\text { Dilatation (AoR, Bentall procedure } 42 \mathrm{y} \text { ), mitral } \\
\text { valve insufficiency, DCM, arrhythmia }\end{array}$ & $\begin{array}{l}\text { Midface hypoplasia, HAP, scoliosis, JH, increased AHR, Ara, EB, delayed wound healing, thin } \\
\text { translucent skin, soft velvety skin, subcutaneous fat markedly reduced }\end{array}$ & ? & $\begin{array}{l}\text { c.517-3_517- } \\
\text { 2delinsAG } \\
\text { p.? }\end{array}$ & LP (I) \\
\hline 7 & IC & q, 10 & $\begin{array}{l}\text { Mitral valve prolapse and insufficiency, billowing } \\
\text { of the tricuspid valve }\end{array}$ & $\begin{array}{l}\text { Height }+1.5 \mathrm{SD} \text {, retrognathia, long face, hypertelorism, DPF, prominent eyes, HAP, scoliosis, JH, } \\
\text { Ara, PP, camptodactyly, hammertoes, thin translucent skin, subcutaneous fat markedly } \\
\text { reduced, decreased muscle mass }\end{array}$ & $\begin{array}{l}\text { De } \\
\text { novo }\end{array}$ & $\begin{array}{l}\text { c.889A }>\text { G } \\
\text { p.(Arg297Gly) }\end{array}$ & LP (V) \\
\hline 8 & IC & 0,58 & $\begin{array}{l}\text { Dilatation (AAo, AoR), aortic valve insufficiency, } \\
\text { IDavid procedure (57y), varices }\end{array}$ & $\begin{array}{l}\text { Height }+3 \text { SD, dolichocephaly, retrognathia, long face, midface hypoplasia, unilateral DPF, HAP, } \\
\text { Ara, subcutaneous fat markedly reduced }\end{array}$ & - & $\begin{array}{l}\text { c.898C }>\text { T } \\
\text { p.(Arg300Trp) }\end{array}$ & $\mathrm{P}(\mathrm{lllb})$ \\
\hline 9 & IC & 0,6 & Mitral valve prolapse & $\mathrm{BU}, \mathrm{PE}, \mathrm{JH}, \mathrm{Ara}$, thoracic kyphosis, foot eversion & $\begin{array}{l}\text { De } \\
\text { novo }\end{array}$ & $\begin{array}{l}\text { c. } 898 C>T \\
\text { p. }(\operatorname{Arg} 300 T r p)\end{array}$ & $\mathrm{P}(\mathrm{IIlb})$ \\
\hline 10 & IC & 0,27 & $\begin{array}{l}\text { Upper normal limit of AoR diameter, mitral valve } \\
\text { insufficiency, moderate dilatation of the left } \\
\text { ventricle }\end{array}$ & $\begin{array}{l}\text { Height +1.5 SD, exotropia, downslanting palpebral fissures, BU, HAP, PC, scoliosis, } \\
\text { dolichostenomelia, Ara, PP, SS, dural ectasia }\end{array}$ & - & $\begin{array}{l}\text { c. } 898 C>T \\
\text { p.(Arg300Trp) }\end{array}$ & $\mathrm{P}(\mathrm{IIlb})$ \\
\hline 11 & IC & $0^{2}, 54$ & $\begin{array}{l}\text { Dissection (type B extending to the AA, } 52 \mathrm{y} \text {, aortic } \\
\text { isthmus diameter at dissection } 71 \mathrm{~mm} \text { ), dilatation } \\
\text { (AoR, 52y) }\end{array}$ & $\begin{array}{l}\text { Height }+1 \text { SD, cataract (bilateral, surgery } 52 \mathrm{y} \text { and } 53 \mathrm{y}) \text {, myopia, BU, pneumothorax }(14 \mathrm{y}), \mathrm{JH} \text {, } \\
\text { PP, hyperelasticity of the skin, subdural hematoma, } \mathrm{IH}(53 \mathrm{y}) \text {, angioneurotic edema }\end{array}$ & + & $\begin{array}{l}\text { c. } 898 \mathrm{C}>\mathrm{T} \\
\text { p.(Arg300Trp) }\end{array}$ & $\mathrm{P}(\mathrm{IIIb})$ \\
\hline 12 & So & 0,14 & Dilatation (AoR, 14y) & Height +0.5 SD, midface hypoplasia, myopia, BU, HAP, PE, increased AHR, Ara, PP & + & $\begin{array}{l}\text { c. } 898 \mathrm{C}>\mathrm{T} \\
\text { p.(Arg300Trp) }\end{array}$ & $\mathrm{P}(\mathrm{lllb})$ \\
\hline 13 & IC &,+ 12 & Mitral valve prolapse & $\begin{array}{l}\text { Height }+0.5 \mathrm{SD} \text {, long face, hypertelorism, blue sclerae, BU, HAP, posterior cleft palate, JH, Ara, } \\
\mathrm{PP}, \mathrm{EB} \text {, delayed wound healing, thin translucent skin, soft velvety skin }\end{array}$ & + & $\begin{array}{l}\text { c.898C }>\mathrm{T} \\
\text { p. }(\operatorname{Arg} 300 \operatorname{Trp})\end{array}$ & $\mathrm{P}(\mathrm{IIlb})$ \\
\hline 14 & Mo & +, 35 & None & $\begin{array}{l}\text { Height }+0.5 \mathrm{SD} \text {, long face, smooth philtrum, hypertelorism, blue sclerae, DPF, HAP, hiatal henia } \\
(18 \mathrm{y}) \text {, JH, increased AHR, Ara, PP, hallux valgus, EB, delayed wound healing, thin translucent } \\
\text { skin, soft velvety skin }\end{array}$ & + & $\begin{array}{l}\text { c.898C }>\mathrm{T} \\
\text { p.(Arg300Trp) }\end{array}$ & $\mathrm{P}(\mathrm{IIIb})$ \\
\hline 15 & $\mathrm{Si}$ & \%, 6 & Mitral valve prolapse & $\begin{array}{l}\text { Height }+1 \text { SD, metopic ridge, retrognathia, long face, hypertelorism, blue sclerae, DPF, ptosis, } \\
\text { HAP, JH, Ara, PP, hallux valgus, EB, delayed wound healing, thin translucent skin, soft velvety } \\
\text { skin }\end{array}$ & + & $\begin{array}{l}\text { c. } 898 \mathrm{C}>\mathrm{T} \\
\text { p.(Arg300Trp) }\end{array}$ & $\mathrm{P}(\mathrm{IIIb})$ \\
\hline 16 & $\mathrm{MU}$ &,+ 40 & Mitral valve prolapse and insufficiency & $\begin{array}{l}\text { Height }+2.3 \mathrm{SD} \text {, long face, hypertelorism, flat cornea, } \mathrm{PC} \text {, osteoarthritis, kyphoscoliosis, JH, } \\
\text { Ara, PP, patellar tendon rupture, fatigue, EB, thin translucent skin, soft velvety skin, skin } \\
\text { hyperextensibility }\end{array}$ & + & $\begin{array}{l}\text { c.898C }>\text { T } \\
\text { p.(Arg300Trp) }\end{array}$ & $P(I I l b)$ \\
\hline
\end{tabular}
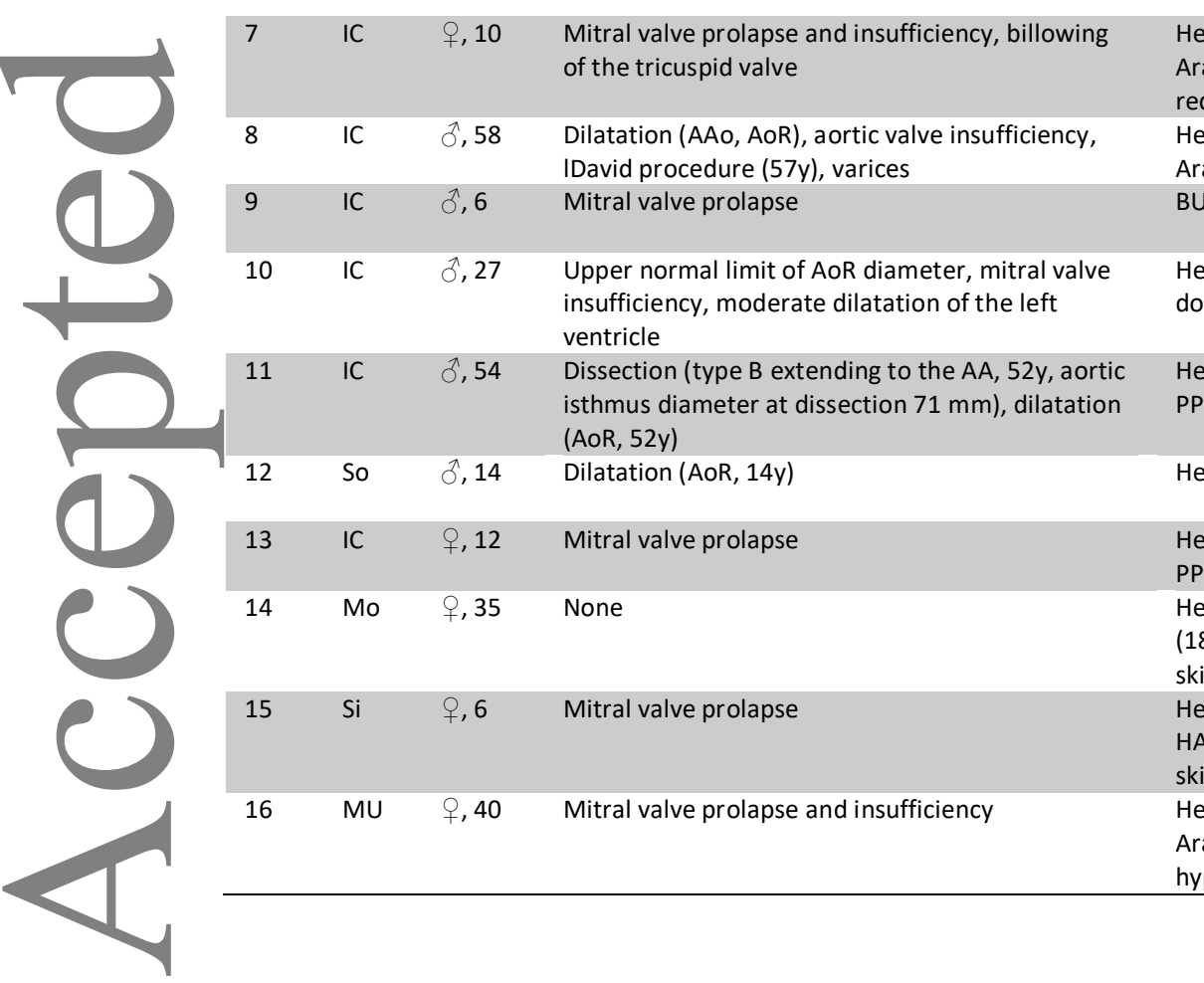


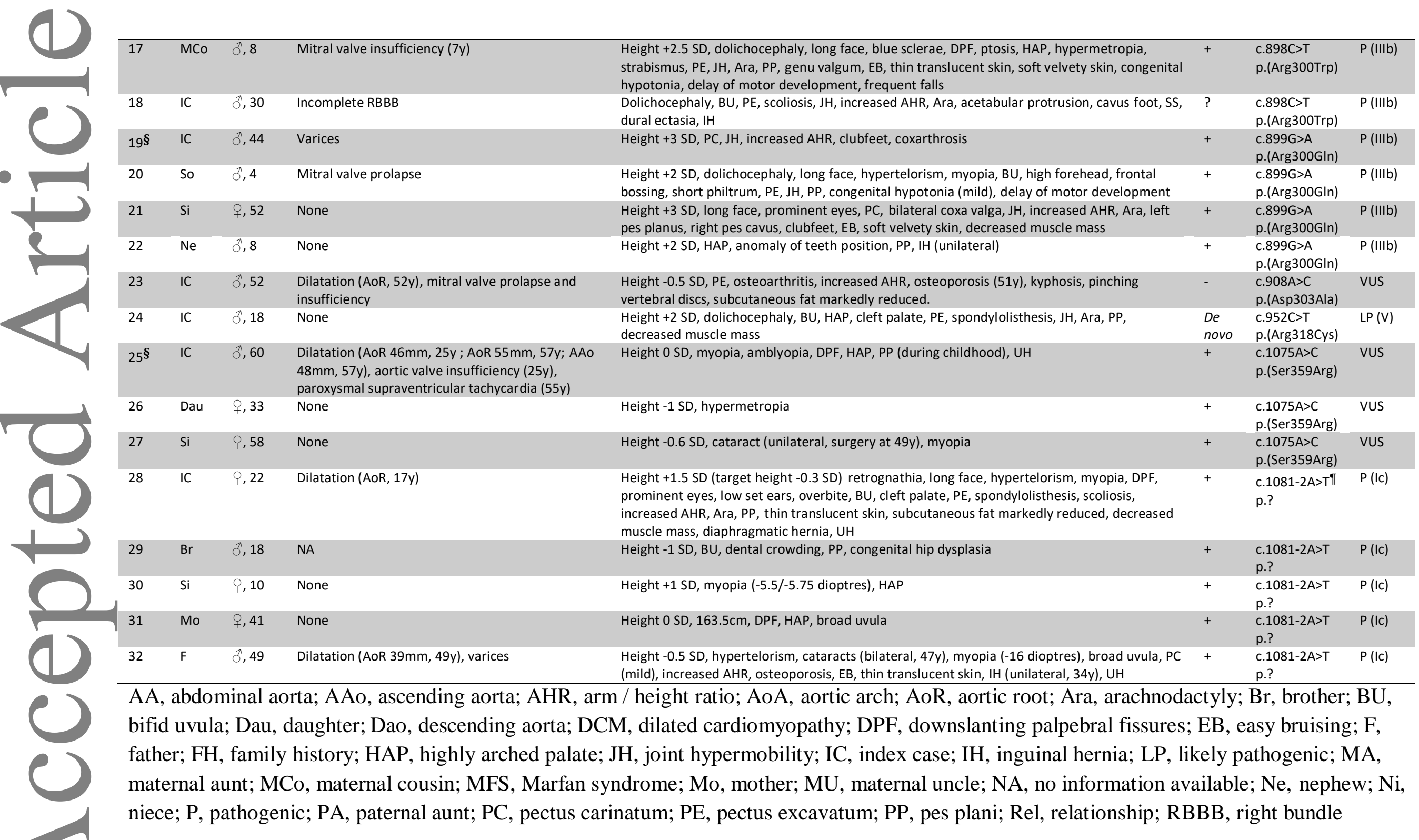


branch block; Rel, relationship; SD, standard deviation; Si, sister; So, son; SS, skin striae; UH, umbilical hernia; VUS, variant of unknown significance; $y$, age at diagnosis (years).

Used TGFB3 RefSeq transcripts (based on Genome build: GRCh37/hg19): NC_000014.8(NM_003239.4)

† Age (in years) at last evaluation

‡ According to the AMCG criteria (Richard et al., 2015)

$\S$ This patient is recently described in literature (Overwater et al., 2018)

ฯ Homozygote

+ known family history

- no family history

? unknown

Table 2. Overview of all reported TGFB3 disease-causing variants

\begin{tabular}{|c|c|c|c|c|}
\hline Nucleotide change & Protein change & Domain & $\begin{array}{l}\text { gnomAD } \\
\text { allele count }\end{array}$ & Reference(s) \\
\hline c.106A>T & p.(Lys36*) & LAP & 0 & Schepers et al., 2018 \\
\hline c.170dup & p.(Glu58*) & LAP & 0 & Current study \\
\hline c.321dup & p.(Phe108llefs*18) & LAP & 0 & ClinVar \\
\hline c. $353-1 G>C$ & p.? & LAP & 0 & ClinVar \\
\hline c.427A $>T$ & p. (Arg143*) & LAP & 0 & Ziganshin et al., 2015 \\
\hline c.437del & p.(Leu146Hisfs*68) & LAP & 0 & Schepers et al., 2018 \\
\hline c. $463 \mathrm{C}>\mathrm{T}$ & p.(Arg155Trp) & LAP & 0 & Current study \\
\hline c. $516+1 G>A$ & p.? & LAP & 0 & Current study \\
\hline c.517-3_517-2delinsAG & p.? & LAP & 0 & Current study \\
\hline c. $517-1 \mathrm{G}>\mathrm{C}$ & p.? & LAP & 0 & ClinVar \\
\hline c.704del & p.(Asn235Metfs*11) & LAP & 0 & Bertoli-Avella et al., 2015 \\
\hline c. $754+2 \mathrm{~T}>\mathrm{C}$ & p.Glu216_Lys251del & LAP & 0 & Bertoli-Avella et al., 2015 \\
\hline c. $787 \mathrm{G}>\mathrm{C}$ & p.(Asp263His) & LAP & 0 & Bertoli-Avella et al., 2015; Schepers et al., 2018 \\
\hline c. $796 \mathrm{C}>\mathrm{T}^{\dagger}$ & p.(Arg266Cys) & LAP & 3 & Schepers et al., 2018 \\
\hline c. $826 \mathrm{C}>\mathrm{T}$ & p.(Pro276Ser) & LAP & 0 & ClinVar \\
\hline c.883_884del & p.(Gly295Serfs*28) & LAP & 1 & ClinVar \\
\hline c. $889 \bar{A}>\mathrm{G}$ & p.(Arg297Gly) & RKKR motif & 0 & Current study \\
\hline c. $898 \mathrm{C}>\mathrm{T}$ & p. (Arg300Trp) & RKKR motif & 0 & Bertoli-Avella et al., 2015; Schepers et al. 2018 \\
\hline
\end{tabular}




\begin{tabular}{|c|c|c|c|c|}
\hline & & & & Current study \\
\hline c. $898 \mathrm{C}>\mathrm{G}$ & p.(Arg300Gly) & RKKR motif & 0 & Kuechler et al., 2015 \\
\hline c. $899 \mathrm{G}>\mathrm{A}$ & p.(Arg300GIn) & RKKR motif & 0 & $\begin{array}{l}\text { Matyas et al., 2014; Kim et al., 2017; Schepers et al., } \\
\text { 2018; Overwater et al., 2018; Current study }{ }^{\prime}\end{array}$ \\
\hline c. $908 \mathrm{~A}>\mathrm{C}^{+}$ & p.(Asp303Ala) & Cytokine & 4 & $\begin{array}{l}\text { 2018; Overwater et al., 2018; Current study } \\
\text { Current study }\end{array}$ \\
\hline c.916del & p. (Tyr306Thrfs*63) & Cytokine & 0 & ClinVar \\
\hline c. $927-1 \mathrm{G}>\mathrm{C}$ & p.? & Cytokine & 1 & ClinVar \\
\hline c. $952 C>T$ & p.(Arg318Cys) & Cytokine & 0 & Current study \\
\hline c. $965 \mathrm{~T}>\mathrm{C}$ & p.(Ile322Thr) & Cytokine & 2 & Bertoli-Avella et al., 2015 \\
\hline c.979G $>\mathrm{T}$ & p.(Asp327Tyr) & Cytokine & 1 & Schepers et al., 2018 \\
\hline c. $989 \mathrm{G}>\mathrm{A}$ & p. $(\operatorname{Trp} 330 *)$ & Cytokine & 0 & ClinVar \\
\hline c.1020T>A & p.(Tyr340*) & Cytokine & 0 & ClinVar \\
\hline c. $1034 C>G$ & p.(Ser345*) & Cytokine & 0 & ClinVar \\
\hline c. $1075 \mathrm{~A}>\mathrm{C}+$ & p.(Ser359Arg) & Cytokine & 0 & Overwater et al., 2018; Current study $\ddagger$ \\
\hline c. $1081-2 A>T^{5}$ & p.? & Cytokine & 0 & Current study \\
\hline c. $1095 C>A$ & p.(Tyr365*) & Cytokine & 0 & Bertoli-Avella et al., 2015 \\
\hline c.1102 $1105 \mathrm{del}$ & p.(Leu368Thrfs*18) & Cytokine & 0 & ClinVar \\
\hline c.1157del & p. (Leu386Argfs*21) & Cytokine & 0 & Bertoli-Avella et al., 2015 \\
\hline c. $1195 \mathrm{G}>\mathrm{T}$ & p.(Glu399*) & Cytokine & 0 & ClinVar \\
\hline c. $1202 T>C$ & p.(Leu401Pro) & Cytokine & 0 & Bertoli-Avella et al., 2015; Schepers et al., 2018 \\
\hline c. $1226 \mathrm{G}>\mathrm{A}$ & p.(Cys409Tyr) & Cytokine & 0 & Rienhoff et al., 2013 \\
\hline
\end{tabular}

Used TGFB3 RefSeq transcript (GRCh37/hg19): NC_000014.8(NM_003239.4)

$\dagger$ Variant of unknown significance

‡ Same patient is reported by Overwater et al., 2018 and in the current study

$\S$ Index case is homozygote 


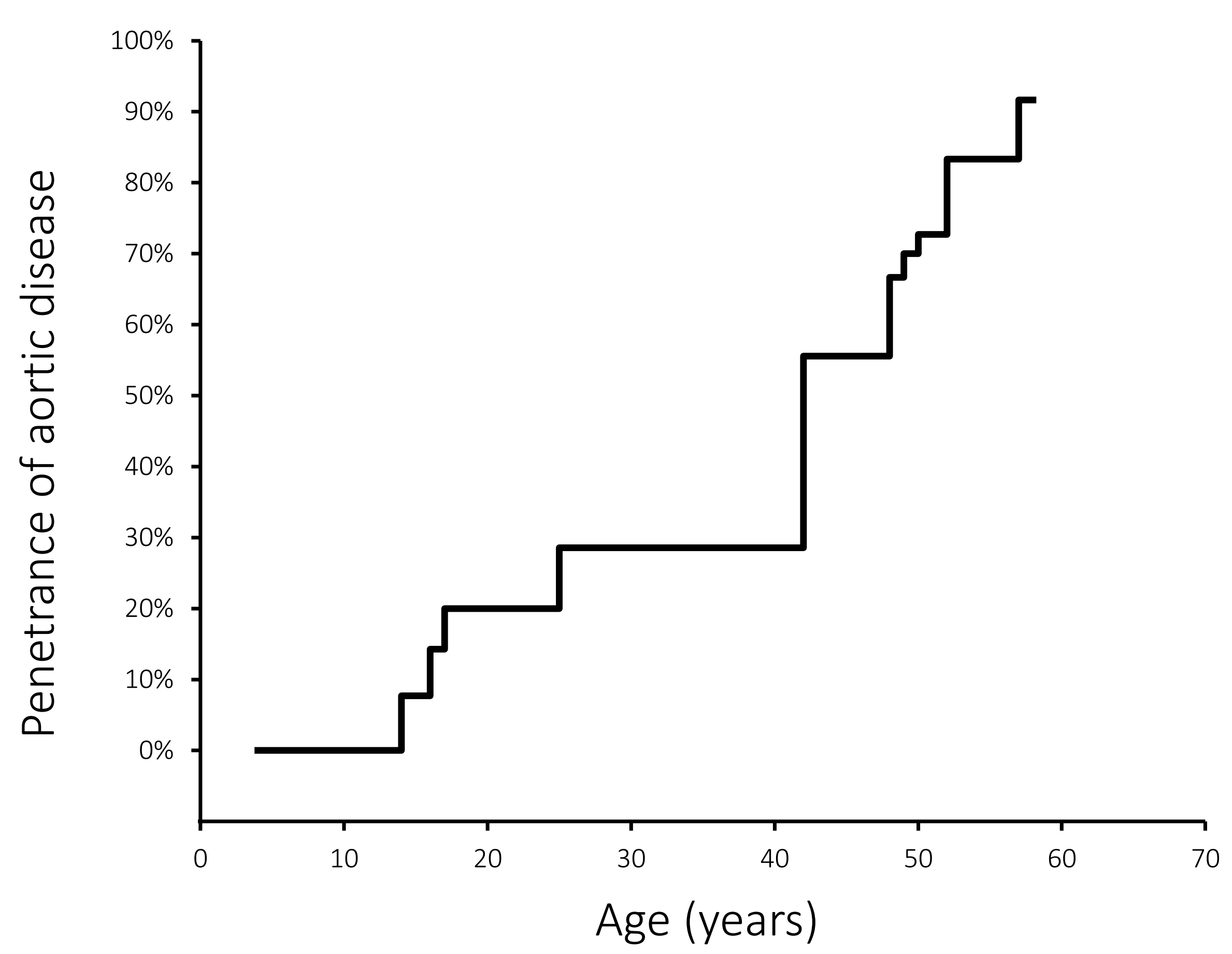




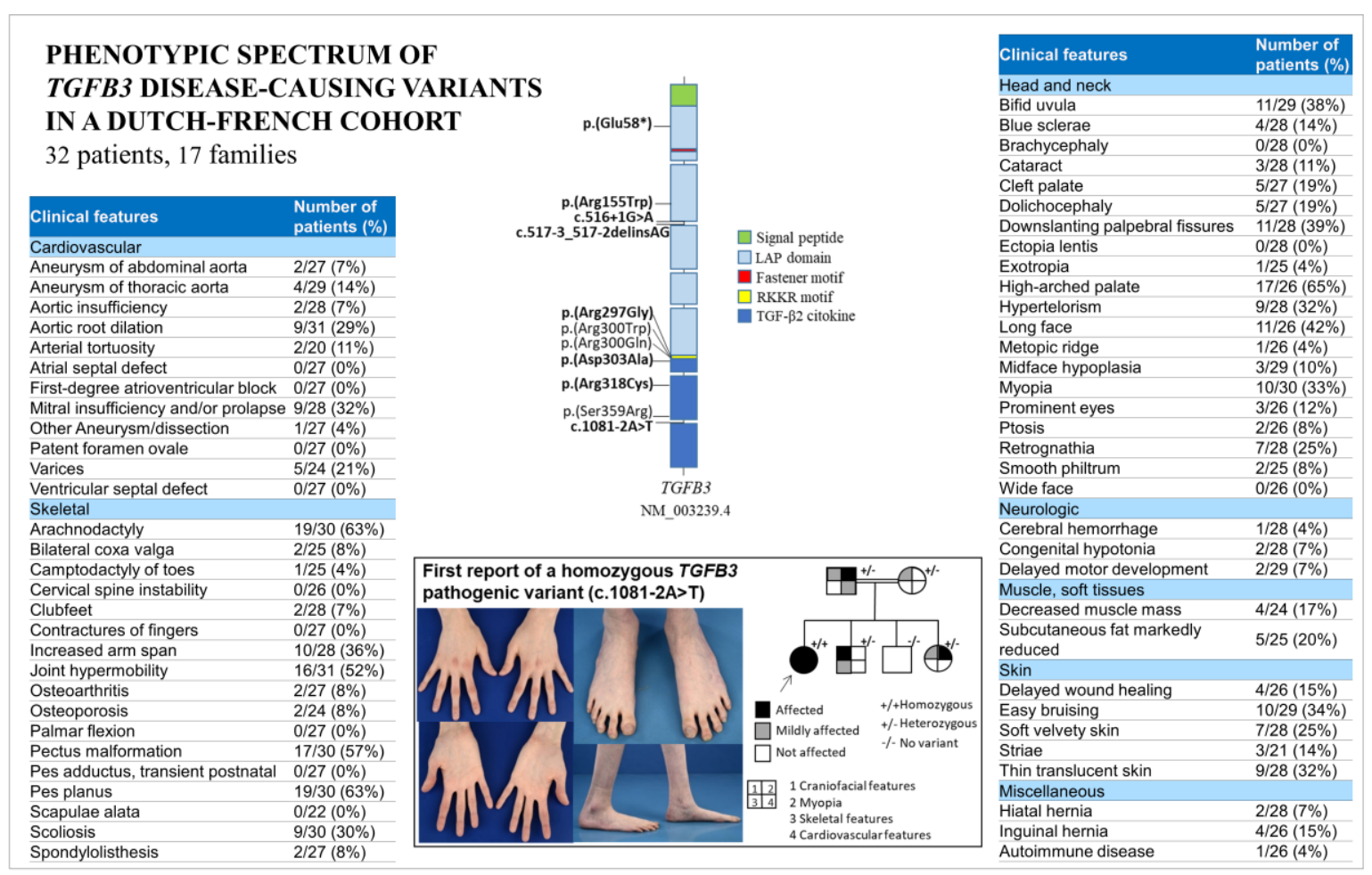

